\title{
Anaphylaxis Induced by Sugammadex in a Patient with Papillary Serous Carcinoma of the Uterine Adnexa Undergoing Exploratory Laparotomy
}

\author{
Allan R. Escher Jr. ${ }^{1}$, Jonathan B. Cohen ${ }^{1}$ \\ 1. Anesthesiology, H. Lee Moffitt Cancer Center and Research Institute, Tampa, USA
}

Corresponding author: Allan R. Escher Jr., allan.escher@moffitt.org

\begin{abstract}
In this case report, we present a patient who developed anaphylaxis immediately after sugammadex administration. A 67-year-old female with the diagnosis of papillary serous carcinoma was scheduled for an exploratory laparotomy exam under anesthesia and total abdominal hysterectomy. At the end of the operation, sugammadex was administered; it was rapidly accompanied with marked hypotension and bradycardia.
\end{abstract}

Multiple boluses of phenylephrine were administered with minimal effect. Boluses of epinephrine and vasopressin were given and the patient's hemodynamic instability rapidly abated. A 12-

lead electrocardiogram (ECG) obtained in the post-anesthesia care unit (PACU) showed sinus tachycardia and a prolonged corrected QT (QTc) interval. A tryptase level was drawn in the operating room. After an uneventful PACU stay, the patient was observed overnight in the intensive care unit (ICU) as a precaution. Cardiology service was consulted, and they agreed with the anesthesia team that the cause of the patient's hemodynamic instability collapse was consistent with the diagnosis of anaphylaxis. The serum tryptase returned with a level of $62.3 \mathrm{ng} / \mathrm{mL}$, confirming the diagnosis. The patient was discharged on postoperative Day 4.

Anaphylaxis may result from sugammadex usage, and this might cause severe hypotension and cardiac arrhythmias. The prompt recognition and treatment of hypotension and anaphylaxis are critical to minimize morbidity and prevent mortality in these patients.

Received 12/22/2018

Review began 12/28/2018 Review ended 01/09/2019 Published 01/12/2019

() Copyright 2019 Escher et al. This is an open access article distributed under the terms of the Creative Commons Attribution License CC-BY 3.0., which permits unrestricted use, distribution, and reproduction in any medium, provided the original author and source are credited.
Categories: Anesthesiology, General Surgery, Oncology

Keywords: anaphylaxis, arrhythmia, hypotension, tryptase, sugammadex, iatrogenic anaphylaxis, medication allergy, adverse drug reactions, epinephrine, adrenaline

\section{Introduction}

Synthetic cyclodextrin derivatives, such as sugammadex, show promise as a safe and well-tolerated agent for the reversal of non-depolarizing neuromuscular blocking agents and the prevention of residual blockade [1]. It is the most recent attempt by the pharmaceutical industry to formulate the "ideal reversal agent." Such an agent would have a rapid onset, be able to provide reversal for a range of neuromuscular blockades, be inexpensive, and be safe when used as directed. Sugammadex seems to satisfy most of these characteristics and incorporates a novel mechanism of action. It encapsulates rocuronium and other aminosteroid neuromuscular blocking agents (NMBAs), facilitating the swift reversal of neuromuscular blockade. As opposed to traditional reversal agents, such as neostigmine, sugammadex can also provide reversal from deep blockade [1]. This ability to provide rapid reversal of s neuromuscular blockade without the side-effects of neostigmine has led to its rapid adoption in practice in the United States, European Union, and Japan.

In Japan, it has been in use since 2010; a three-year retrospective study revealed an incidence of sugammadex-induced anaphylaxis at $0.039 \%$ ( $n=6$; $95 \%$ confidence interval (CI), 0.014\%-0.084\%), which could be comparable to that of succinylcholine or rocuronium [2]. Following the withdrawal of high-profile medications, such as rofecoxib, aprotinin, and rapacuronium, clinicians have a good reason for caution with adopting new medications into routine clinical practice. In fact, these concerns slowed the introduction of sugammadex in the United States until 2015, when it received approval from the US Food and Drug Administration (FDA) [3]. We report a case of anaphylaxis following sugammadex administration in a patient with papillary serous ovarian carcinoma. The patient had no history of hypersensitivity to sugammadex. In addition, she had received seven cycles of neoadjuvant therapy with carboplatin and paclitaxel prior to surgery.

\section{Case Presentation}

A 67 -year-old, $115.3 \mathrm{~kg}, 157-\mathrm{cm}$ female patient with papillary serous adenocarcinoma of the uterine adnexa and uterine masses was brought to the operating room for an exam under anesthesia, exploratory laparotomy, total abdominal hysterectomy, bilateral salpingo-oophorectomy, omentectomy, debulking, and 
lymph node dissection. Her medical history included hypertension, hemorrhoids, morbid obesity (body mass index (BMI) 46.8), and a pulmonary embolism three months prior to surgery. Enoxaparin sodium was stopped two days prior to surgery. The patient's functional capacity was moderate to excellent at greater than or equal to four metabolic equivalents (METS). She reported completion of all household chores, cycled three to five miles daily and was capable of climbing two flights of stairs. She reported no history of anesthetic complications and no family history of anesthetic complications. Her surgical history included one previous colonoscopy, for which she received deep sedation.

The patient's home medications included prochlorperazine $10 \mathrm{mg}$ as needed after chemotherapy, dexamethasone $4 \mathrm{mg}$ for three days only after chemotherapy, rivaroxaban $20 \mathrm{mg}$ daily, and enoxaparin 120 $\mathrm{mg} / 0.8 \mathrm{~mL}$ solution subcutaneous (held two days prior to surgery). In addition, hydrochlorothiazidevalsartan 12.5-320 mg was taken by the patient daily for control of essential hypertension. A non-smoker, the patient reported one alcoholic beverage an average of four times per month. Her medication allergies consisted of benazepril (tachycardia), ezetimibe (myalgias), simvastatin (myalgias), and triamterene (myalgias).

Premedication for anxiolysis was accomplished with $2 \mathrm{mg}$ midazolam. In the operating room, standard anesthesia monitors were applied, including non-invasive blood pressure measurement, electrocardiogram, peripheral oxygen saturation, and a temperature-sensing Foley catheter. Her baseline vital signs were a temperature of 36.94 degrees Celsius, blood pressure $138 / 80 \mathrm{~mm} \mathrm{Hg}$, heart rate 81 beats/min, and oxygen saturation $\left(\mathrm{SaO}_{2}\right)$ 99\%. Induction of general anesthesia proceeded with the administration of $180 \mathrm{mg}$ of $1 \%$ propofol, $70 \mathrm{mg}$ lidocaine, and $50 \mathrm{mcg}$ fentanyl after preoxygenation with $100 \%$ oxygen. Tracheal intubation was facilitated with the administration of $40 \mathrm{mg}$ rocuronium. A $7.0 \mathrm{~mm}$ cuffed oral endotracheal tube was placed with one attempt and then secured at $22 \mathrm{~cm}$. The maintenance of anesthesia with sevoflurane was initiated and the patient received $10 \mathrm{mg}$ of intravenous (IV) dexamethasone after the start of surgery. Mechanical ventilation with volume control was selected with $6 \mathrm{~mL} / \mathrm{kg}$ and a fraction of inspired oxygen $\left(\mathrm{FiO}_{2}\right)$ of 0.5 . A second $18 \mathrm{~g}$ IV was started in the left hand and an arterial line was placed in the right radial artery with one attempt.

At the conclusion of the surgery, $400 \mathrm{mg}$ IV sugammadex ( $3.74 \mathrm{mg} / \mathrm{kg}$ ) was given as reversal for the neuromuscular blockade (the train-of-four was 0/4). Within 60 seconds, her blood pressure dropped to 58/39 $\mathrm{mm} \mathrm{Hg}$, heart rate remained stable at 80 , and $\mathrm{SpO}_{2}$ dropped to $94 \%$. Phenylephrine was titrated in $0.2 \mathrm{mg}$ doses for a total of $1 \mathrm{mg}$. Intravenous fluids were administered liberally and the surgical team notified of intractable hypotension. Ischemic changes were observed in electrocardiogram (ECG) leads II and V at the same time that the heart rate began to increase. Given the presentation of symptoms in close proximity to the administration of sugammadex, the possibility of anaphylaxis was considered. At this time, $150 \mathrm{mcg}$ IV epinephrine was given followed an additional dose of $100 \mathrm{mcg}$. Vasopressin two units IV was then administered to restore the blood pressure to baseline in the setting of epinephrine-induced tachycardia. Auscultation revealed clear breath sounds and there was no increase in airway pressures noted. However, a maculopapular rash was noted over the upper thorax. Greater attention was given to the possibility of anaphylaxis at this point and diphenhydramine $50 \mathrm{mg}$ and famotidine $20 \mathrm{mg}$ were administered. A serum tryptase level was sent to the laboratory to assist with determining the cause of the patient's symptoms. After her condition was stabilized, the patient was successfully extubated and transferred to the recovery room. A 12-lead electrocardiogram showed sinus tachycardia and a prolongation of the corrected QT (QTc) interval (Figure 1). 


\section{Cureus}

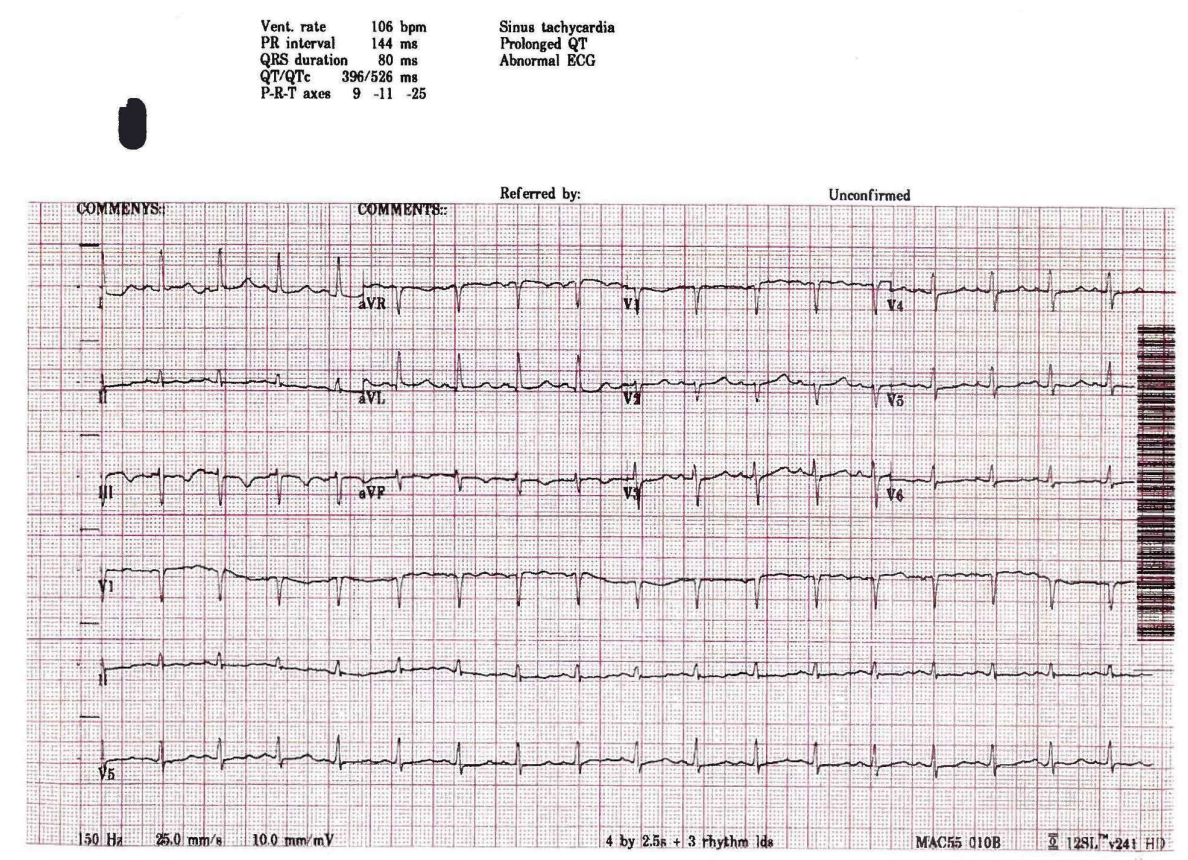

\section{FIGURE 1: ECG upon admission to PACU}

ECG: electrocardiogram

PACU: post-anesthesia care unit

QTc: corrected QT interval

QT: time from start of the $Q$ wave to end of the T wave

Vent: ventricular

PR: time from start of $\mathrm{p}$ wave to start of the QRS complex

Cardiology was consulted and the patient was observed in the intensive care unit overnight as a precaution. Dermatological manifestations resolved within a few hours. Serum tryptase from the time of the episode returned at $62 \mathrm{ng} / \mathrm{mL}$ (normal < $11.4 \mathrm{ng} / \mathrm{mL}$ ), which effectively confirms that the cause of this patient's symptoms was consistent with an anaphylactic reaction. She was discharged home on postoperative Day 4 with a recommendation for follow-up with her primary care physician.

\section{Discussion}

The introduction of sugammadex has led to its widespread use in Europe, Japan, and the United States, and it has demonstrated safety in patients with pulmonary disease at the $2 \mathrm{mg} / \mathrm{kg}$ and $4 \mathrm{mg} / \mathrm{kg}$ dosing regimens [4]. However, a recent study showed hypersensitivity can occur in healthy subjects without a history of prior exposure $[3,5]$. Data on the epidemiology of perioperative anaphylaxis is variable, with an incidence ranging from $1 / 1,250$ to $1 / 18,600$ per administration [6]. This wide range reflects the difficulty of determining precisely what factors may predispose a patient to hypersensitivity or anaphylaxis. Both in vivo (i.e. skin tests) and in vitro (i.e. basophil activation tests) have demonstrated positive test results with sugammadex $[7]$.

The challenge in the operating room is the myriad of medications that our patients are commonly exposed to, which may result in anaphylaxis: neuromuscular blocking agents (NMBAs), antibiotics, blood products, dyes, and latex [8]. In the case at hand, symptoms manifested within 60 seconds of drug administration. In a prior study, signs of anaphylaxis appeared in 14 out of 15 patients, with a mean time of 1.9 minutes ( $1 \mathrm{~min}$ and $52 \mathrm{~s}$ ) after a dose of sugammadex [9]. In this case, the placement of an arterial line facilitated the prompt recognition of hypotension.

The rapid administration of epinephrine remains the intervention of first choice because of its alpha-1 agonist vasoconstrictor effects, beta-1 agonist chronotropic and inotropic effects, and beta-2 agonist effects [9]. A 25-year retrospective review of anaphylaxis mortality showed only $23 \%$ of individuals received epinephrine before cardiac arrest [10]. Mortality from iatrogenic anaphylaxis improved, with $42 \%$ of patients 
receiving epinephrine prior to cardiac arrest [10]. It is helpful to understand the risk factors that may predispose a patient to anaphylaxis from neuromuscular blocking agents.

A retrospective review of 2,022 cases of NMBA hypersensitivity revealed 1,247 cases of severe NMBAinduced anaphylaxis and 84 fatalities (4.1\%) [11]. A multivariate analysis concluded the risk factors associated with a fatal outcome: male gender, emergency surgery, a history of hypertension or another cardiovascular disease, obesity, and ongoing beta-blocker treatment [11]. Our patient had the following risk factors: hypertension, a history of pulmonary embolus, and obesity.

Serum tryptase is an important marker of mast cell activation; levels may peak after one to two hours and then decline with a t $1 / 2$ of 1.5 to 2 hours [12]. The specimen should be obtained rapidly in cases of suspected anaphylaxis. Additionally, clear communication with our surgical colleagues should occur when a further neuromuscular blockade is requested, particularly at the end of the case when the risks and benefits can be addressed [13].

\section{Conclusions}

It is important to remember that perioperative anaphylaxis can occur in healthy patients without a prior history of hypersensitivity to NMBAs. The prompt recognition of perioperative anaphylaxis is critical since symptoms may present suddenly. Epinephrine remains the first-line treatment for perioperative anaphylaxis, to reduce morbidity and mortality. Additionally, the clinician should be aware of the risk factors for mortality from the use of NMBAs: male gender, emergency surgery, hypertension or cardiovascular disease, obesity, and ongoing beta-blocker therapy. Serum tryptase levels should be obtained in order to help clarify the clinical picture, although the results will not be immediately available at the point of care. Finally, the re-administration of neuromuscular blocking agents should take place with interdisciplinary communication and collaboration, especially when re-dosing occurs at the end of a case.

\section{Additional Information \\ Disclosures}

Human subjects: Consent was obtained by all participants in this study. Conflicts of interest: In compliance with the ICMJE uniform disclosure form, all authors declare the following: Payment/services info: All authors have declared that no financial support was received from any organization for the submitted work. Financial relationships: All authors have declared that they have no financial relationships at present or within the previous three years with any organizations that might have an interest in the submitted work. Other relationships: All authors have declared that there are no other relationships or activities that could appear to have influenced the submitted work.

\section{Acknowledgements}

Conception and design of the article: AE Acquisition of data: AE, JC Drafting of manuscript: AE, JC

\section{References}

1. Nag K, Singh D, Shetti A, Kumar H, Sivashanmugam T, Parthasarathy S: Sugammadex: a revolutionary drug in neuromuscular pharmacology. Anesth Essays Res. 2013, 7:302-306. 10.4103/0259-1162.123211

2. Miyazaki Y, Sunaga H, Kida K, Hobo S, Inoue N, Muto M, Uezono S: Incidence of anaphylaxis associated with sugammadex. Anesth Analg. 2018, 26:1505-1508. 10.1213/ANE.0000000000002562

3. Savic L, Savic S, Hopkins PM: Sugammadex: the sting in the tail?. Br J Anaesth. 2018, 121:694-697. 10.1016/j.bja.2018.07.014

4. Amao R, Zornow M, Cowan R, Cheng D, Morte J, Allard M: Use of sugammadex in patients with a history of pulmonary disease. J Clin Anesth. 2011, 24:289-297. 10.1016/j.jclinane.2011.09.006

5. Min KC, Bondiskey P, Schulz V, et al.: Hypersensitivity incidence after sugammadex administration in healthy subjects: a randomised controlled trial. Br J Anaesth. 2018, 121:749-757. 10.1016/j.bja.2018.05.056

6. Mertes PM, Volcheck GW, Garvey LH, Takazawa T, Platt P, Guttormsen A, Tacquard C: Epidemiology of perioperative anaphylaxis. Presse Med. 2016, 45:758-767. 10.1016/j.lpm.2016.02.024

7. Orihara M, Takazawa T, Saito S: Is sugammadex alone sufficient to cause anaphylaxis? . J Anesth. 2018, 32:307. 10.1007/s00540-018-2453-4

8. Simons FER, Ebisawa M, Sanchez-Borges M, et al.: 2015 update of the evidence base: World Allergy Organization anaphylaxis guidelines. World Allergy Organ J. 2015, 8:32. 10.1186/s40413-015-0080-1

9. Tsur A, Kalansky A: Hypersensitivity associated with sugammadex administration: a systematic review . Anaesthesia. 2014, 69:1251-1257. 10.1111/anae.12736

10. Xu YS, Kastner M, Harada L, Xu A, Salter J, Waserman S: Anaphylaxis-related deaths in Ontario: a retrospective review of cases from 1986 to 2011. Allergy Asthma Clin Immunol. 2014, 10:1-8. 10.1186/17101492-10-38

11. Reitter M, Petitpain N, Latarche C, et al.: Fatal anaphylaxis with neuromuscular blocking agents: a risk factor and management analysis. Eur Ann Allergy Clin Immunol. 2014, 69:954-959. 10.1111/all.12426

12. Schwartz L, Yunginger J, Miller J, Bokhari R, Dull D: Time course of appearance and disappearance of human mast cell tryptase in the circulation after anaphylaxis. J Clin Invest. 1989, 83:1551-1555. 10.1172/JCI114051

13. Answine Joseph F, Lamberg James J: Interdisciplinary intraoperative communication and collaboration 


\section{Cureus}

needed for optimal neuromuscular blockade management. J Anaesthesiol Clin Pharmacol. 2014, 30:434-444. 10.4103/0970-9185.137302 